\title{
Further Reading and References
}

Beck, K.: Color atlas of laparoscopy (Saunders, Philadelphia

1984). Becker, CD.; Jameson, M.; Fache, J.S.; Burhenne, H.J.: Catheter for

endoluminal bipolar electrocoagulation. Radiology 170: 561-

562(1989). Becker, CD.; Quenville, N.F.; Burhenne, H.J.: Long-term occlusion

of the porcine cystic duct by means of endoluminal radio-frequency electrocoagulation.

Radiology 767: 63-68 (1988). Becker, CD.; Quenville, N.F.; Burhenne, H.J.: Gallbladder

ablation

through radiologic intervention: an experimental alternative to

cholecystectomy. Radiology 171: 235-240 (1989). Berci, G.; Cuschieri, A.: Practical laparoscopy

(Baillière Tindall,

London 1986). Berci, G.; Hamlin, J.A.: Operative biliary radiology (Williams \&

Wilkins, Baltimore 1981). Berci, G.; Morgenstem, L.; Shore, J.M., et al.: A direct approach to the different diagnosis of jaundice. Am. J. Surg. 726: 372-374

(1973). Berci, G.; Paz-Partlow, M.: Electronic imaging in endoscopy. Surg.

Endosc. 2: 227-233 (1988). Boyle, W.S.; Smith, G.E.: Charged couple semiconductor devices.

Bell Syst. Tech. J. 49: 285-290 (1970). Buess, G.: Endoskopische Mikrochirurgie - minimally invasive surgery. Endoskopie Heute 7: 30-43 (1988). Buess, G.; Mentges, B.; Melzer, A.; Gutt, C; Schafer, D.; Nikisch,

N.: Laparoscopic microsurgical cholecystostomy in gallstone

disease (Abstract). Surg. Endosc. 4 : 44 (1990). Burhenne, H.J.; Stoller, J.L.:

Minicholecystostomy and radiologic

stone extraction in high risk cholelithiasis patients. Am. J. Surg.

149: 632-635 (1985). Christensen, T.; Bendix, T.; Kehlet, H.: Fatigue and cardiorespira-

tory function following abdominal surgery. Br. J. Surg. 69: 417-

419(1983). Cope, C: Percutaneous subhepatic cholecystostomy with removable

anchor. Am. J. R. 757: 1129-1132 (1988). Cranley, N.; Logan, H.: Exploration of the common

bile duct - the

relevance of the clinical picture and the importance of preoper-

ative cholangiography. Br. J. Surg. 67: 869 (1980). Cuschieri, A.: Value of laparoscopy in general surgery and gastroen-

terology. J. Hosp. Med. 24: 252-257 (1980). Cuschieri, A.: A laparoscopic revolution. J. R. Coll. Surg. Educ. 34:

295 (1990). Cuschieri, A.; Berci, G.: Laparoscopic biliary surgery (Blackwell

Scientific Publications, Oxford 1990).

Cuschieri, A.; Berci, G.; McSherry, C: Editorial opinion: laparoscopic cholecystectomy. Am. J. Surg. 759: 273 (1990).

Cuschieri, A.; El Ghany, A.B.Abd.; Holley, M.P.: Successful chemical cholecystectomy: a laparoscopic guided technique. Gut 30: 1786-1794(1989). 
Dawson, J.L.: Cholecystectomy, in Smith of Marlow, Sherlock, Surgery of the gall bladder and bile ducts; 2nd ed., p. 313 (Butter-worth, London 1981).

Del Santo, P.; Kaxarian, K.K.; Rogers, J.F.; Bevins, P.A.; Hall, J.R.: Prediction of operative cholangiography in patients undergoing elective cholecystectomy with routine liver function chemistries. Surgery 98:1 (1985).

Den Besten, L.; Berci, G.: The current status of biliary tract surgery: an international study of 1072 consecutive patients. Wld J. Surg. 10: 116(1986).

Ding, Z.; Rutten, H.; Jackimowicz, J.J.: The evaluation of selection for ESWL of gallstones. J. dig. Surg. (in press).

Doyle, P.J.; Ward-McQuaid, J.N.; McEwen-Smith, E.: The value of routine peroperative cholangiography - a report of 4000 chole-cystectomies. Br. J. Surg. 69: 617 (1982).

Dubois, F.; Berthelot, G.; Levard, H.: Cholécystectomie par cælio-scopie. Presse méd. 18: 980982 (1989).

Dubois, F.; et al.: Coelioscopic cholecystectomy. Ann. Surg. 277: 60-62 (1990).

Eggermont, A.M.; Lameris, J.S.; Jeekel, J.: Ultrasound-guided percutaneous transhepatic cholecystostomy for acute acalculous cholecystitis. Arch. Surg. 720: 1354-1357 (1985).

El Ghany, A.B.Abd.; Holley, M.P.; Cuschieri, A.: Percutaneous clearance of the gallbladder through an access cholecystectomy: a laparoscopic guided technique. Surg. Endosc. 3: 126-130 (1989).

Esallon, A.; Rosales, W.; Aldrete, J.S.: Reliability of pre- and intra-operative tests for biliary lithiasis. Ann. Surg. 207: 640 (1985).

Feste, J.R.: Laser laparoscopy. J. reprod. Med. 30: 413-417 (1985).

Fleming, J.S.: Laparoscopically directed appendicectomy. Aust. N.Z. J. Obstet. Gynaec. 25: 238239 (1985).

Gacetta, D.J.; Cohen, M.J.; Crummy, A.B.; Joseph, D.V.; Kuglitsch, M.; Mack, E.: Ultrasonic lithotripsy of gallstones after cholecystostomy. Am. J. R. 143: 1088-1089 (1984).

Getrajdman, G.I.; O’Toole, K.; Laffey, K.J.; Martin, E.C: Cystic duct occlusion and transcatheter sclerosis of the gallbladder in the rabbit. Investve Radiol. 27: 400-403 (1986).

Getrajdman, G.I.; O’Toole, K.; Logerfo, P.; Laffey, K.J.; Martin,

134

Further Reading and References

E.C.: Transcatheter sclerosis of the gallbladder in rabbits. A preliminary study. Investve Radiol. 20: 393-398 (1985).

Gibney, R.G.; Fache, J.S.; Becker, CD.; et al.: Combined surgical and radiologic intervention for complicated cholelithiasis in high risk patients. Radiology 165: 715-719 (1987).

Glenn, F.: Importance of the technique in cholecystectomy. Surgery Gynec. Obstet. 101: 201

(1955).

Gomel, V.: Operative laparoscopy: time for acceptance. Fert. Steril. 52: 1-11 (1989).

Götz, F.: Die endoskopische Appendektomie nach Semm bei der akuten und chronischen Appendizitis. Endoskopie Heute 2: 5-7 (1988).

Götz, F.; Pier, A.; Bacher, C: Modified laparoscopic appendectomy in surgery. Surg. Endosc. 4: 6-9 (1990).

Greiner, L.; Wenzel, H.; Jakobeit, C.H.: Biliäre Stosswellen-Litho-trypsie. Fragmentation und Lyse - ein neues Verfahren. Dt. med. Wschr. 112: 1893-1896 (1987). 
Hajiro, K.; Matsui, H.; Tsujimura, T.: Endoscopic hemostasis with hemoclips, local injection and other new techniques. Endoscopy, suppl., pp. 62-67(1986).

Ham, F.M.: Cholecystectomy; in Blumgart, Surgery of the liver and biliary tract, pp. 559-569 (Churchill Livingstone, London 1988).

Hauer-Jensen, M.; Karesen, R.; Nygaard, K.; Solheim, K.; Amlie, E.; Havig, O.; Viddal, K.O.: Predictive ability of cholecystoli-thiasis indicators - a prospective evaluation. Ann. Surg. 202: 64 (1985).

Hess, W.: Die Cholezystektomie; in Hess, Rohner, Cirenei, Akovbi-antz, Die Erkrankungen der Gallenwege und des Pankreas, vol. 2, pp. 1409-1419 (Piccin, Padova 1986).

Inui, K.; Nakazawwa, S.; Kimoto, E.; Naito, Y.; Yamao, K.: Non-surgical treatment of cholelithiasis with percutaneous transhe-patic cholecystocopy. Am. J. Gastroenterol. 83: 11241127 (1988).

Irving, A.D.; Cuschieri, A.: Laparoscopy in the jaundiced patient. A review of 53 patients. Br. J. Surg. 65: 678-680 (1978).

Jakimowicz, J.J.: Intraoperative ultrasound-biliary disease; in Blumgart, Surgery of the liver and biliary tract (Churchill Livingstone, London 1988).

Jakimowicz, J.J.; Carol, E.J.; Jürgens, P.; Rutten, H.; Sommeling, C; Tielbeek. A.: Intraoperative ultrasonography: an alternative to intraoperative cholangiography. Medicamundi 32/1: 35-39 (1987).

Jackimowicz, J.J.; Carol, E.J.; Mak, B.; Roukema, A.: An operative choledochoscopy the flexible choledochoscope. Surgery Gynec. Obstet. 162: 215-221 (1986).

Jakimowicz, J.J.; Rutten, H.; Jürgens, Ph.J.; Carol, E.J.: Comparison of operative ultrasonography and radiography in screening of the common bile duct for calculi. Wld J. Surg. 11: 628-634 (1987).

Jakimowicz, J.J.; Rutten, H.; Sommeling, C; Ding, Z.: Intraoperative ultrasonography of the biliary tract, equipment, technique and results; in Brinkman, Strosche, Stellenwert der Sonographie in der Chirurgie, pp. 89-97 (TM Verlag, Hamelen 1990).

Jennett, B.: High technology medicine, benefits and burdens, pp. 227-248 (Oxford University Press, London 1986).

Jolly, P.C.; Baker, J.W.; Schmidt, H.M.; Walker, J.H.; Holm, J.C.: Operative cholangiography: a case for its routine use. Ann. Surg. 765:551 (1968).

Kellet, M.J.; Wickham, J.E.A.; Russell, R.C.G.: Percutaneous chole-cystolithotomy. Br. med. J. 296: 453-455 (1988).

Kerlan, R.K.; LaBerge, J.M.; Ring, E.J.: Percutaneous cholecystoli-thotomy: preliminary experience. Radiology 157: 653-656 (1985).

Laffey, K.J.; Martin, E.C.: Percutaneous removal of large gallstones. Gastrointest. Radiol. 11: 165-168 (1986).

Leahy, P.F.: Technique of laparoscopic appendicectomy. Br. J. Surg. 76: 616 (1989).

Lee, P.H.; Hopkins, T.B.; Howard, P.J.: Percutaneous cholecystoli-thotomy. Urology 18: 37-39 (1989).

Lindemann, S.R.; Tung, G.; Silverman, S.G.; Mueller, P.R.: Percutaneous cholecystostomy. A review. Semin. Intervent. Radiol. 5: 179-185(1988).

Lohela, P.; Soiva, M.; Suo, I.; Taavitsainen, M.; Holopainen, O.: Ultrasonic guidance for percutaneous puncture and drainage in acute cholecystitis. Acta radiol. (Diag.) 27: 543-546 (1986). 
McKinlay, J.B.: From 'promising report' to 'standard procedure': seven stages in the career of a medical innovation. Milbank meml Fund 59: 374-411 (1981).

Mills, J.L.; Beck, D.E.; Harford, F.J.: Routine operative cholangiography. Surgery Gynec. Obstet. 161: 343 (1985).

Mofti, A.B.; Ahmed, I.; Tandon, R.C.; Al-Tameem, M.M.; Al-Khu-dairy, N.N.: Routine or selective preoperative cholangiography. Br. J. Surg. 73 : 548 (1986).

Paul, A.; Troidl, H.; Gay, K.; Viell, B.; Bode, C: Hilft die Cholezystektomie? Chirurg (in press). Paumgartner, G.: Fragmentation of gallstones by extracorporeal shock wave. Semin. Liver Dis. 7: 317 (1987).

Paumgartner, G.: Therapy of stone disease: fragmentation. Postgraduate Course AGA, New Orleans 1988.

Paumgartner, G.: The Munich experience with biliary lithotripsy. Second Interdisciplinary International Symposium on Biliary Lithotripsy, Vancouver 1989.

Paz-Partlow, M.: Documentation for laparoscopy; in Berci, Cuschieri, Practical laparoscopy, pp. 19-32 (Baillière Tindall, London 1986).

Pearse, D.M.; Hawkins, I.F.; Shaver, R.; Vogel, S.: Percutaneous cholecystostomy in acute cholecystitis and common duct obstruction. Radiology 152: 365-367 (1984).

Perrisat, J.; Collet, D.; Belliard, R.: Gallstones: laparoscopic treatment - cholecystectomy, cholecystostomy, and lithotripsy. Our own experience. Surg. Endosc. 4: 1-5 (1990).

Popp, L.W.: Endoscopic patch repair of inguinal hernia in a female patient. Surg. Endosc. 4: 1012 (1990).

Reddick, E.J.; Olsen. D.O.: Laparoscopic laser cholecystectomy. A comparison with mini-lap cholecystectomy. Surg. Endosc. 3: 131-133(1989).

Reddick, E.; Olsen, D.O.; Daniell, J.J.; et al.: Laparoscopic laser cholecystectomy. Laser Med. Surg. News Adv. 7: 38-40 (1989).

Remley, K.B.; Cubberley, D.A.; Wanatabe, A.S.; Nelson, J.A.; Colby, T.V.: Systemic absorption of gallbladder sclerosing agents in the rabbit. A preliminary study. Investve Radiol. 21: 396-399 (1986).

Röder, H.: Die Technik der Mandelgesundungsbestrebungen. (A description of the loop for tonsillectomy in children.) Ärztl. Rdsch. 57: 169-171 (1918).

Rösch, W.: Reizmagen - was tun? Dt. Ärzteblatt 87 36: 29-35 (1990).

Sackmann, M.; Delius, M.; Sauerbruch, T.; Holl, J.; Weber, W.; Ippisch, E.; Hagelauer, U; Wess, O.; Hepp, W.; Brendel, E.;

Further Reading and References

135

Paumgartner, G.: Shock-wave lithotripsy of gallbladder stones: the first 175 patients. New Engl. J. Med. 318: 393-398 (1988).

Salomonowitz, E.; Frick, M.P.; Simmons, R.L.; O’Leary, J.F.; Lund, G.; Cragg, A.H.; Amplatz, K.: Obliteration of the gallbladder without formal cholecystectomy. A feasibility study. Archs Surg. 199: 725-729 (1984).

Sauerbruch, T.; Delius, M.; Paumgartner, G.; Holl, J.; Wess, O.; Weber, W.; Hepp, W.; Brendel, W.: Fragmentation of gallstones by extracorporeal shock waves. New Engl. J. Med. 314: 818822 (1986).

Seif, R.M.: Routine operative cholangiography: a critical appraisal. Am. J. Surg. 134: 566 (1977). 
Semm, K.: Endocoagulation: a new field of endoscopic surgery. J. reprod. Med. 16: 175-180 (1976).

Semm, K.: Tissue-puncher and loop-ligation. New aids for surgical-therapeutic pelviscopy (laparoscopy) = endoscopic intraabdomi-nal surgery. Endoscopy 10: 119-124 (1978).

Semm, K.: Endoscopic appendicectomy. Endoscopy 75: 59-64 (1983).

Semm, K.: Operative manual for endoscopic abdominal surgery (Year Book, Chicago 1987).

Semm, K.; Mettler, L.: Technical progress in pelvic surgery via operative laparoscopy. Am. J. Obstet. Gynec. 138: 121-127 (1980).

Shaver, R.W.; Hawkins, I.F.; Soong, J.: Percutaneous cholecystosto-my. Am. J. R. 138: 11331136 (1982).

Sigel, B.; et al.: Comparative accuracy of operative ultrasonography and cholangiography in detecting common bile duct calculi. Surgery 94: 715-720 (1983).

Spangenberger, W.; Klein, J.; Troidl, H.: Laparoskopische Cholezy-stektomie - erste

Erfahrungen und Ergebnisse. Arch. klin. Chir. 2: 1361-1368(1990).

Stark, M.E.; Loughry, C.W.: Routine operative cholangiography with cholecystectomy. Surgery Gynec. Obstet. 757: 657 (1980).

Talley, N.J.; McNeil, D.; Piper, D.W.: Discriminant value of dyspeptic symptoms: a study of the clinical presentation of 221 patients with dyspepsia of unknown cause, peptic ulceration and cholelithiasis. Gut 28: 40-46 (1987).

Troidl, H.; Neugebauer, E.: Akuter Schmerz in der Chirurgie - Kli-nische Bedeutung, Messmethoden und Therapie. Chirurg 67: 485-493 (1990).

Troidl, H.; Paul, A.: Cholelithiasis und Cholezystitis. Therapiewo-che 36: 3320-3327 (1986).

Troidl, H.; Spangenberger, W.; Dietrich, A.; Neugebauer, E.: Laparoskopische

Cholezystektomie, erste Erfahrungen und Ergebnisse bei 300 Operationen: eine prospektive Beobachtungsstu-die. Chirurg (in press).

Troidl, H.; Spangenberger, W.; Kusche, J.: Bewertung der Endosko-pie. Arch. klin. Chir. 2: 385392 (1988).

Van Sonnenberg, E.; Wittich, G.R.; Gasola, G.; et al.: Diagnostic and therapeutic percutaneous gallbladder procedures. Radiology 760: 23-26 (1986).

Vogelzang, R.L.; Memcek, A.A.: Percutaneous cholecystostomy: diagnostic and therapeutic efficacy. Radiology 765: 29-34 (1988).

Wannagat, L.: Laparoskopische Cholangiographie. Radiology 13: 26-34(1973).

Warren, K.W.: Technique of cholecystectomy and choledochotomy. Surg. Clins N. Am. 36: 687 (1956).

Warren, L.P.; Kadir, S.; Dunnick, N.R.: Percutaneous cholecystostomy: anatomic considerations. Radiology 168: 615-616 (1988).

Wood-Dauphinée, Sh.; Troidl, H.: Endpoints for clinical studies: conventional and innovative variables; in Troidl, Spitzer, McPeek, Mulder, McKneally, Principles and practice of research (Springer, Berlin 1986). 